\title{
Asymptotic Analysis for One-Name Credit Derivatives
}

\author{
Yong-Ki Ma ${ }^{1}$ and Beom Jin Kim ${ }^{2}$ \\ ${ }^{1}$ Department of Applied Mathematics, Kongju National University, Chungcheongnam-Do 314-701, Republic of Korea \\ ${ }^{2}$ Department of Mathematics, Yonsei University, Seoul 120-749, Republic of Korea
}

Correspondence should be addressed to Beom Jin Kim; beomjinkim@yonsei.ac.kr

Received 1 March 2013; Accepted 16 May 2013

Academic Editor: Carlos Vazquez

Copyright (C) 2013 Y.-K. Ma and B. J. Kim. This is an open access article distributed under the Creative Commons Attribution License, which permits unrestricted use, distribution, and reproduction in any medium, provided the original work is properly cited.

We propose approximate solutions to price defaultable zero-coupon bonds as well as the corresponding credit default swaps and bond options. We consider the intensity-based approach of a two-correlated-factor Hull-White model with stochastic volatility of interest rate process. Perturbations from the stochastic volatility are computed by using an asymptotic analysis. We also study the sensitive properties of the defaultable bond prices and the yield curves.

\section{Introduction}

It is well known that the methodology for modeling a credit risk can be split into two primary approaches of models that attempt to describe default processes (see Duffie and Singleton [1] and Bielecki and Rutkowski [2] for general references): the structural approach such as those developed by Merton [3], Longstaff and Schwartz [4], Leland and Toft [5], Zhou [6], Duffie and Lando [7], Hilberink and Rogers [8], and Giesecke [9] and the intensity-based approach such as those developed by Jarrow and Turnbull [10], Madan and Unal [11], Lando [12], Duffie and Singleton [13], and CollinDufresne and Goldstein [14]. A structural approach assumes that the market has complete information with respect to the underlying firm's value process and capital structure. In contrast, an intensity-based approach (also known as a reduced-form approach) has been developed where default is determined as the first jump of an exogenously given jump process. Hence, an underlying firm's default time is inaccessible and driven by a default intensity, a function of some latent state variables. Since we are concerned with modeling the default time, we adopt the intensity-based approach with the fractional recovery assumption of Duffie and Singleton [13].

Since the initial contribution to the intensity-based approach is given by Jarrow and Turnbull [10], who consider a constant and a deterministic Poisson intensity, there has been many mathematical studies on credit risk. Lando [12] uses that the default process is described by a Cox process. Schönbucher [15] develops the term structure model of defaultable interest rates by using the Heath-Jarrow-Morton model, and Tchuindjo [16] studies the price of a defaultable zero-coupon bond with two-correlated-factor Hull-White model. Duffee [17] and Driessen [18] perform the estimation of an intensity-based model using a Kalman filter approach, and Bayraktar and Yang [19] propose intensity-based unified models with stochastic volatility calibrated to stock options data. Some of other application articles based on an intensitybased framework are as follows. Duffie and Gârleanu [20] extend the single-name intensity setting to a multiname setting by putting firms' default intensities as the sum of an idiosyncratic factor and a common factor that affects the default of all firms. Papageorgiou and Sircar [21] study the pricing for the prices of single-name credit derivatives such as defaultable bonds, options on defaultable bonds, and credit default swaps (CDSs) using a function of two different time-scale intensity models. In particular, Ma and Kim [22] provide a pricing formula for the CDSs based on modeling the intensity as a jump-diffusion process.

The main contribution of this paper is as follows. Tchuindjo [16] studies the two-correlated-factor Hull-White model to propose a closed-form solution to price the defaultable bonds, supposing a nonzero correlation between interest rate process and intensity process. However, Tchuindjo's 
model does not appear hump-shaped yield curve that matches a typical yield curve for the defaultable bonds as in Merton's model [3] (see Figure 2). Cotton et al. [23] also show the bursty nature of stochastic volatility of interest rate process to understand the effect of uncertain and changing volatility on interest rate derivatives. So, we expand the twocorrelated-factor Hull-White model by modifying constant volatility of interest rate process and use an asymptotic analysis for prices of the defaultable zero-coupon bonds. Our numerical results indicate that the defaultable zero-coupon bonds with stochastic volatility of interest rate process affects both quantitative and qualitative effect. We also obtain formulas for CDSs and options when the underlying assets are defaultable zero-coupon bonds.

This paper is structured as follows. In Section 2, we obtain a partial differential equation (PDE) based on stochastic volatility of interest rate process to get the price of the defaultable zero-coupon bonds. In Section 3, an approximate solution to price defaultable zero-coupon bonds is derived by using an asymptotic analysis including some numerical examples. Section 4 applies the results of Section 3 to the CDSs and the bond options. In Section 5, we give the final concluding remarks.

\section{Defaultable Zero-Coupon Bonds}

In this section, we consider an intensity-based approach to price the defaultable zero-coupon bonds with stochastic volatility of the interest rate process.

2.1. Constant Volatility of Interest Rate Process. The framework is the intensity-based approach with the fractional recovery assumption of Duffie and Singleton [13] as follows:

$$
\mathscr{Z}(t, T)=\mathbb{E}^{*}\left[e^{-\int_{t}^{T}\left\{r_{s}+(1-R) \lambda_{s}\right\} d s} \mid \mathscr{F}_{t}\right],
$$

under a risk-neutral probability $P^{*}$, where $t$ is a current time, $T$ is an expiry time, $R$ is a recovery rate with $R \in[0,1]$, and $\mathscr{F}_{t}$ is a filtration generated by the joint process of interest rate process $r_{t}$ and intensity process $\lambda_{t}$. We need to work out the expectation over the possible paths of $r_{t}$ and $\lambda_{t}$ to get the defaultable zero-coupon bond prices.

We review the price of the defaultable zero-coupon bonds when the interest rate process and the intensity process are correlated and each of these processes follows a Hull-White model [24]. Under a risk-neutral probability $P^{*}$, we adopt the Tchuindjo type of stochastic differential equations (SDEs),

$$
\begin{gathered}
d r_{t}=\left(\theta_{t}-a r_{t}\right) d t+\sigma d W_{t}^{(r) *} \\
d \lambda_{t}=\left(\tilde{\theta}_{t}-\tilde{a} \lambda_{t}\right) d t+\widetilde{\sigma} d W_{t}^{(\lambda) *} \\
d W_{t}^{(r) *} d W_{t}^{(\lambda) *}=\rho d t
\end{gathered}
$$

for such models, where $a, \tilde{a}, \sigma$, and $\widetilde{\sigma}$ are constants, $\theta_{t}$ and $\tilde{\theta}_{t}$ are time-varying deterministic functions, and $W_{t}^{(r) *}$ and $W_{t}^{(\lambda) *}$ are correlated Brownian motions. Under the fractional recovery of market value assumption, the PDE for the price of the defaultable zero-coupon bond is derived. Then, this PDE is analytically solved. Refer to Tchuindjo [16].

2.2. Stochastic Volatility of Interest Rate Process. In this subsection, we enlarge the constant volatility of the interest rate process by incorporating the stochastic volatility of the interest rate process.

We replace the constant $\sigma$ in the SDE (2) by a stochastic process $\sigma_{t}$ given by a smooth, bounded below and above, and strictly positive function $f\left(y_{t}\right)$ of an Ornstein-Uhlenbeck (OU) process $y_{t}$ as follows (see Fouque et al. [25]):

$$
d y_{t}=\alpha\left(m-y_{t}\right) d t+\beta d W_{t}^{(y) *},
$$

where $\alpha$ is a rate of meanreversion of volatility, $\beta$ is a volatility of volatility, and $W_{t}^{(y) *}$ is a Brownian motion in a risk-neutral probability $P^{*}$. Note that the OU process is an ergodic process with a unique invariant distribution, which is a Gaussian distribution with mean $m$ and variance $v^{2}=\beta^{2} / 2 \alpha$. For the asymptotic analysis, $\alpha$ driving the volatility is large, and we are interested in approximation in the limit $\alpha \rightarrow \infty$ with $\nu^{2}$ remaining constant. In this OU model, therefore, we set

$$
\alpha=\frac{1}{\epsilon}, \quad \beta=\mathcal{O}\left(\frac{1}{\sqrt{\epsilon}}\right), \quad \nu=\mathcal{O}(1),
$$

where $\epsilon$ is a small and strictly positive parameter. So, in the risk-neutral probability $P^{*}$ the joint process $\left(r_{t}, \lambda_{t}, y_{t}\right)$ is given by the following SDEs:

$$
\begin{gathered}
d r_{t}=\left(\theta_{t}-a r_{t}\right) d t+\sigma_{t} d W_{t}^{(r) *}, \\
\sigma_{t}=f\left(y_{t}\right), \\
d y_{t}=\frac{1}{\epsilon}\left(m-y_{t}\right) d t+\frac{\nu \sqrt{2}}{\sqrt{\epsilon}} d W_{t}^{(y) *}, \\
d \lambda_{t}=\left(\tilde{\theta}_{t}-\tilde{a} \lambda_{t}\right) d t+\widetilde{\sigma} d W_{t}^{(\lambda) *},
\end{gathered}
$$

where the standard Brownian motions $W_{t}^{(r) *}, W_{t}^{(\lambda) *}$, and $W_{t}^{(y) *}$ are dependent of each other such that the correlation structure is given by

$$
\begin{aligned}
& d\left\langle W^{(r) *}, W^{(\lambda) *}\right\rangle_{t}=\rho_{r \lambda} d t, \\
& d\left\langle W^{(r) *}, W^{(y) *}\right\rangle_{t}=\rho_{r y} d t, \\
& d\left\langle W^{(\lambda) *}, W^{(y) *}\right\rangle_{t}=\rho_{\lambda y} d t .
\end{aligned}
$$

The defaultable zero-coupon bond prices with a fractional recovery rate $R$ at time $t$ for an interest rate process $r_{t}=r$, an intensity process $\lambda_{t}=\lambda$, and a stochastic volatility process $y_{t}=y$, denoted by $P(t, r, \lambda, y ; T)$, are given by

$$
\begin{aligned}
& P(t, r, \lambda, y ; T) \\
& \quad=\mathbb{E}^{*}\left[e^{-\int_{t}^{T}\left\{r_{s}+(1-R) \lambda_{t}\right\} d s} \mid r_{t}=r, \lambda_{t}=\lambda, y_{t}=y\right],
\end{aligned}
$$


and then, using the three-dimensional Feynman-Kac formula, we have the Kolmogorov PDE:

$$
\begin{aligned}
& \frac{\partial P}{\partial t}+\left(\theta_{t}-a r\right) \frac{\partial P}{\partial r}+\left(\tilde{\theta}_{t}-\tilde{a} \lambda\right) \frac{\partial P}{\partial \lambda}+\frac{1}{\epsilon}(m-y) \frac{\partial P}{\partial y} \\
& +\frac{1}{2} f^{2}(y) \frac{\partial^{2} P}{\partial r^{2}}+\frac{1}{2} \widetilde{\sigma}^{2} \frac{\partial^{2} P}{\partial \lambda^{2}}+\frac{\nu^{2}}{\epsilon} \frac{\partial^{2} P}{\partial y^{2}} \\
& +\rho_{r \lambda} \tilde{\sigma} f(y) \frac{\partial^{2} P}{\partial r \partial \lambda}+\rho_{r y} f(y) \frac{\nu \sqrt{2}}{\sqrt{\epsilon}} \frac{\partial^{2} P}{\partial r \partial y} \\
& +\rho_{\lambda y} \widetilde{\sigma} \frac{\nu \sqrt{2}}{\sqrt{\epsilon}} \frac{\partial^{2} P}{\partial \lambda \partial y}-(r+(1-R) \lambda) P=0
\end{aligned}
$$

with the terminal condition $\left.P(t, r, \lambda, y ; T)\right|_{t=T}=1$. Refer to Øksendal [26].

\section{Asymptotic Analysis}

In this section, we provide the main results of the paper. We present an asymptotic analysis to the solution of the PDE (8) and give an approximate solution of the defaultable zerocoupon bond price for $\epsilon$.

The PDE (8) is rewritten as follows:

$$
\begin{gathered}
\mathscr{L P}(t, r, \lambda, y ; T)=0, \quad t<T, \\
\left.P(t, r, \lambda, y ; T)\right|_{t=T}=1,
\end{gathered}
$$

where

$$
\begin{gathered}
\mathscr{L}:=\frac{1}{\epsilon} \mathscr{L}_{0}+\frac{1}{\sqrt{\epsilon}} \mathscr{L}_{1}+\mathscr{L}_{2}, \\
\mathscr{L}_{0}=(m-y) \frac{\partial}{\partial y}+v^{2} \frac{\partial^{2}}{\partial y^{2}}, \\
\mathscr{L}_{1}=\sqrt{2} f(y) v \rho_{r y} \frac{\partial^{2}}{\partial r \partial y}+\sqrt{2} \widetilde{\sigma} v \rho_{\lambda y} \frac{\partial^{2}}{\partial \lambda \partial y}, \\
\mathscr{L}_{2}=\frac{\partial}{\partial t}+\left(\theta_{t}-a r\right) \frac{\partial}{\partial r}+\left(\widetilde{\theta}_{t}-\tilde{a} \lambda\right) \frac{\partial}{\partial \lambda} \\
+\frac{1}{2} f^{2}(y) \frac{\partial^{2}}{\partial r^{2}}+\frac{1}{2} \widetilde{\sigma}^{2} \frac{\partial^{2}}{\partial \lambda^{2}} \\
+\widetilde{\sigma} f(y) \rho_{r \lambda} \frac{\partial^{2}}{\partial r \partial \lambda}-(r+(1-R) \lambda),
\end{gathered}
$$

respectively. In particular, $\mathscr{L}_{2}$ is the infinitesimal generator of the two-correlated-factor Hull-White model at current level $y$ of the stochastic volatility process $y_{t}$.

Now, we wish to utilize an asymptotic analysis for $P(t, r, \lambda, y ; T)$ in terms of $\epsilon$. We consider an expansion of $P$ in the following form:

$$
P=P_{0}+\sqrt{\epsilon} P_{1}+\epsilon P_{2}+\epsilon \sqrt{\epsilon} P_{3}+\cdots \text {. }
$$

Applying (11) to (9) leads to

$$
\begin{aligned}
\frac{1}{\epsilon} \mathscr{L}_{0} P_{0} & +\frac{1}{\sqrt{\epsilon}}\left(\mathscr{L}_{0} P_{1}+\mathscr{L}_{1} P_{0}\right)+\left(\mathscr{L}_{0} P_{2}+\mathscr{L}_{1} P_{1}+\mathscr{L}_{2} P_{0}\right) \\
& +\sqrt{\epsilon}\left(\mathscr{L}_{0} P_{3}+\mathscr{L}_{1} P_{2}+\mathscr{L}_{2} P_{1}\right)+\cdots=0 .
\end{aligned}
$$

3.1. The Leading Order Term. In this subsection, we derive the leading order term $P_{0}$.

Theorem 1. One supposes $P_{0}$ to have the affine representation

$$
P_{0}(t, r, \lambda ; T)=e^{A(t, T)-B(t, T) r-(1-R) C(t, T) \lambda},
$$

with $A(T, T)=B(T, T)=C(T, T)=0$. Then, $A(t, T), B(t, T)$ and $C(t, T)$ are given by

$$
\begin{aligned}
& A(t, T)= \ln \frac{P_{0}(0, T)}{P_{0}(0, t)}-\left.C(t, T) \frac{\partial}{\partial s} \ln P_{0}(0, s)\right|_{s=t} \\
&-\left.(B(t, T)-C(t, T)) \frac{\partial}{\partial s} \ln \widetilde{\mathscr{E}}(0, s)\right|_{s=t} \\
&+\frac{\widehat{\sigma}^{2}}{4 a^{3}}\left\{\left(e^{-a T}-e^{-a t}\right)^{2}-a^{2} B^{2}(t, T)\right\} \\
&+\frac{\rho_{r \lambda} \widetilde{\sigma} \bar{a}}{a \widetilde{a}(a+\widetilde{a})}(1-R) \\
& \times\{(a+\widetilde{a})(T-t) \\
&+\frac{\widetilde{\sigma}^{2}}{4 \widetilde{a}^{3}}(1-R)\left[\left(e^{-\widetilde{a} T}-e^{-\widetilde{a} t}\right)^{2}-\widetilde{a}^{2} C^{2}(t, T)\right. \\
& \quad-R\{2 \widetilde{a}(T-t) \\
& B(t, T)=\frac{1}{a}\left\{1-e^{-a(T-t)}\right\}, \\
& \quad-(\widetilde{a} C(t, T)+3) \frac{1}{\widetilde{a}}\left\{1-e^{-\widetilde{a}(T-t)}\right\}, \\
&\times(\widetilde{a} C(t, T)-1)-3\}]
\end{aligned}
$$

respectively, where $\bar{\sigma}$ and $\hat{\sigma}^{2}$ are given by

$$
\begin{gathered}
\bar{\sigma}:=\langle f\rangle=\int_{\mathbb{R}} f(y) \phi(y) d y, \\
\widehat{\sigma}^{2}:=\left\langle f^{2}\right\rangle=\int_{\mathbb{R}} f^{2}(y) \phi(y) d y
\end{gathered}
$$

with the probability density function $\phi(y)=(1 /$ $\left.\sqrt{2 \pi v^{2}}\right) \exp \left\{-(y-m)^{2} / 2 v^{2}\right\}$ for $y \in \mathbb{R}$. Here, $\widetilde{\mathscr{Z}}(0, t)$ is the price at time 0 of a default-free zero-coupon maturing at time t under the Hull-White model [24]. 
Proof. Multiplying (12) by $\epsilon$ and then letting $\epsilon$ go to zero, we obtain the first two terms as follows:

$$
\begin{gathered}
\mathscr{L}_{0} P_{0}=0, \\
\mathscr{L}_{0} P_{1}+\mathscr{L}_{1} P_{0}=0,
\end{gathered}
$$

respectively. Since the infinitesimal operator $\mathscr{L}_{0}$ is the generator of the OU process, the solution $P_{0}$ of (17) must be a constant with respect to $y$ variable, namely, $P_{0}=P_{0}(t, r, \lambda ; T)$. Similarly, from (18) we get $\mathscr{L}_{1} P_{0}=0$ since $P_{0}$ does not rely on $y$ variable. So, the solution $P_{1}$ of (18) must be a constant with respect to $y$ variable, namely, $P_{1}=P_{1}(t, r, \lambda ; T)$. Hence, the two terms $P_{0}$ and $P_{1}$ do not depend on the current level $y$ of the stochastic volatility process $y_{t}$. In this method, we can continue to eliminate the terms of order $1, \sqrt{\epsilon}, \epsilon, \ldots$. For the order- 1 term, we get $\mathscr{L}_{0} P_{2}+\mathscr{L}_{1} P_{1}+\mathscr{L}_{2} P_{0}=0$. This PDE becomes

$$
\mathscr{L}_{0} P_{2}+\mathscr{L}_{2} P_{0}=0
$$

since $P_{1}$ does not rely on $y$ variable. This PDE is a Poisson equation for $P_{2}$ with respect to infinitesimal operator $\mathscr{L}_{0}$. It is well known that it has a solution only if $\mathscr{L}_{2} P_{0}$ is centered with respect to the probability density function $\phi(y)$ of the Gaussian distribution with mean $m$ and variance $v^{2}$, namely,

$$
\left\langle\mathscr{L}_{2} P_{0}\right\rangle=\left\langle\mathscr{L}_{2}\right\rangle P_{0}=0
$$

with the terminal condition $\left.P_{0}(t, r, \lambda ; T)\right|_{t=T}=1$, where $\langle\cdot\rangle$, called by the solvability condition, denotes the expectation with respect to invariant distribution of $y_{t}$. Here, $\left\langle\mathscr{L}_{2}\right\rangle$ is an infinitesimal operator given by

$$
\begin{aligned}
\left\langle\mathscr{L}_{2}\right\rangle= & \frac{\partial}{\partial t}+\left(\theta_{t}-a r\right) \frac{\partial}{\partial r}+\left(\widetilde{\theta}_{t}-\tilde{a} \lambda\right) \frac{\partial}{\partial \lambda} \\
& +\frac{1}{2} \widehat{\sigma}^{2} \frac{\partial^{2}}{\partial r^{2}}+\frac{1}{2} \widetilde{\sigma}^{2} \frac{\partial^{2}}{\partial \lambda^{2}} \\
& +\widetilde{\sigma} \bar{\sigma} \rho_{r \lambda} \frac{\partial^{2}}{\partial r \partial \lambda}-(r+(1-R) \lambda),
\end{aligned}
$$

from (10), where $\bar{\sigma}$ and $\widehat{\sigma}^{2}$ are defined by (15) and (16), respectively. Plugging (21) into (13), we have (14) by using the result of Tchuindjo [16].

Note that in the stochastic volatility setting, we get the leading order term $P_{0}$, which is a generalization of the constant volatility result obtained by Tchuindjo. If $f(y)$ is a constant function, then our results (14) reduce to Tchuindjo's result.

3.2. The First Perturbation Term $\widetilde{P}_{1}$. In this subsection, we derive the first perturbation term $P_{1}$ by using the leading order term $P_{0}$.

Theorem 2. One supposes $\widetilde{P}_{1}$ to have the affine representation

$$
\widetilde{P}_{1}(t, r, \lambda ; T)=D(t, T) e^{A(t, T)-B(t, T) r-(1-R) C(t, T) \lambda},
$$

with $A(T, T)=B(T, T)=C(T, T)=D(T, T)=0$. Then, $A(t$, $T), B(t, T), C(t, T)$, and $D(t, T)$ are given by (14) and

$$
\begin{aligned}
D(t, T)=-\int_{t}^{T}[ & K_{1}^{\epsilon}\left\{(1-R)^{2} B(s, T) C^{2}(s, T)\right\} \\
+ & K_{2}^{\epsilon}\left\{(1-R) B^{2}(s, T) C(s, T)\right\} \\
+ & \left.K_{3}^{\epsilon} B^{3}(s, T)\right] d s,
\end{aligned}
$$

respectively.

Proof. The order- $\sqrt{\epsilon}$ terms in (12) lead to $\mathscr{L}_{0} P_{3}+\mathscr{L}_{1} P_{2}+$ $\mathscr{L}_{2} P_{1}=0$ which is a Poisson equation for $P_{3}$ whose solvability condition is given by

$$
\left\langle\mathscr{L}_{1} P_{2}+\mathscr{L}_{2} P_{1}\right\rangle=0 .
$$

From (19) and (20), we get

$$
P_{2}=-\mathscr{L}_{0}^{-1}\left(\mathscr{L}_{2}-\left\langle\mathscr{L}_{2}\right\rangle\right) P_{0}+c(t, r, \lambda),
$$

for some function $c(t, r, \lambda)$. Plugging (25) into (24), we derive a PDE for $P_{1}$ as follows:

$$
\left\langle\mathscr{L}_{2}\right\rangle P_{1}=\left\langle\mathscr{L}_{1} \mathscr{L}_{0}^{-1}\left(\mathscr{L}_{2}-\left\langle\mathscr{L}_{2}\right\rangle\right)\right\rangle P_{0},
$$

with the terminal condition $\left.P_{1}(t, r, \lambda ; T)\right|_{t=T}=0$. Since we focus on the first perturbation term to $P_{0}$, we reset (26) with respect to $\widetilde{P}_{1}:=\sqrt{\epsilon} P_{1}$ as follows:

$$
\left\langle\mathscr{L}_{2}\right\rangle \widetilde{P}_{1}=\mathscr{A} P_{0}
$$

where $\mathscr{A}$ is given by $(1 / \sqrt{\alpha})\left\langle\mathscr{L}_{1} \mathscr{L}_{0}^{-1}\left(\mathscr{L}_{2}-\left\langle\mathscr{L}_{2}\right\rangle\right)\right\rangle$. From (10) and (21), we have

$$
\begin{aligned}
\mathscr{L}_{2}-\left\langle\mathscr{L}_{2}\right\rangle= & \frac{1}{2}\left(f^{2}(y)-\left\langle f^{2}\right\rangle\right) \frac{\partial^{2}}{\partial r^{2}} \\
& +\rho_{r \lambda} \tilde{\sigma}(f(y)-\langle f\rangle) \frac{\partial^{2}}{\partial r \partial \lambda} .
\end{aligned}
$$

Here, we introduce that the functions $\theta: \mathbb{R} \rightarrow \mathbb{R}$ and $\psi:$ $\mathbb{R} \rightarrow \mathbb{R}$ are given by the solutions of

$$
\mathscr{L}_{0} \theta=f^{2}(y)-\left\langle f^{2}\right\rangle, \quad \mathscr{L}_{0} \psi=f(y)-\langle f\rangle,
$$

respectively, and hence, we get the operator $\mathscr{A}$ denoted by

$$
\mathscr{A}=K_{1}^{\epsilon} \frac{\partial^{3}}{\partial r \partial \lambda^{2}}+K_{2}^{\epsilon} \frac{\partial^{3}}{\partial r^{2} \partial \lambda}+K_{3}^{\epsilon} \frac{\partial^{3}}{\partial r^{3}},
$$

where $K_{1}^{\epsilon}, K_{2}^{\epsilon}$, and $K_{3}^{\epsilon}$ are defined by

$$
\begin{gathered}
K_{1}^{\epsilon}=\frac{\sqrt{2} \nu}{\sqrt{\alpha}} \widetilde{\sigma}^{2} \rho_{r \lambda}\left\langle\psi^{\prime}\right\rangle, \\
K_{2}^{\epsilon}=\frac{v}{\sqrt{2 \alpha}} \widetilde{\sigma} \rho_{\lambda y}\left\langle\theta^{\prime}\right\rangle+\frac{\sqrt{2} \nu}{\sqrt{\alpha}} \widetilde{\sigma} \rho_{r \lambda} \rho_{r y}\left\langle f \psi^{\prime}\right\rangle, \\
K_{3}^{\epsilon}=\frac{\nu}{\sqrt{2 \alpha}} \rho_{r y}\left\langle f \theta^{\prime}\right\rangle,
\end{gathered}
$$


respectively. Hence, we obtain the PDE (27) as follows:

$$
\begin{aligned}
& \frac{\partial \widetilde{P}_{1}}{\partial t}+\left(\theta_{t}-a r\right) \frac{\partial \widetilde{P}_{1}}{\partial r}+\left(\widetilde{\theta}_{t}-\widetilde{a} \lambda\right) \frac{\partial \widetilde{P}_{1}}{\partial \lambda}+\frac{1}{2} \widehat{\sigma}^{2} \frac{\partial^{2} \widetilde{P}_{1}}{\partial \lambda^{2}} \\
&+ \frac{1}{2} \widetilde{\sigma}^{2} \frac{\partial^{2} \widetilde{P}_{1}}{\partial r^{2}}+\bar{\sigma} \breve{\sigma} \rho_{r \lambda} \frac{\partial^{2} \widetilde{P}_{1}}{\partial r \partial \lambda}-(r+(1-R) \lambda) \widetilde{P}_{1} \\
&=-\left[K_{1}^{\epsilon}\left\{B(t, T)(1-R)^{2} C^{2}(t, T)\right\}\right. \\
&\left.+K_{2}^{\epsilon}\left\{B^{2}(t, T)(1-R) C(t, T)\right\}+K_{3}^{\epsilon} B^{3}(t, T)\right] P_{0},
\end{aligned}
$$

with the terminal condition $\left.\widetilde{P}_{1}(t, r, \lambda ; T)\right|_{t=T}=0$. So, plugging (22) into (32), we obtain the result of Theorem 2 by direct computation.

Therefore, synthesizing Theorems 1 and 2, we derive an asymptotic analysis of the defaultable zero-coupon bond prices at time $t$ which is given by

$$
P(t, r, \lambda, y ; T) \approx(1+D(t, T)) e^{A(t, T)-B(t, T) r-(1-R) C(t, T) \lambda},
$$

where $A(t, T), B(t, T), C(t, T)$, and $D(t, T)$ are given by (14), and (23), respectively.

3.3. Numerical Results. In this subsection, we show effects of the first perturbation term with some sensitive analyses of the model parameters. Note that the main numerical implication of the asymptotic analysis is reduction of the parametric dependence of the price formula. Refer to Cotton et al. [23].

We calculate the magnitude of mispricing with respect to defaultable zero-coupon bonds as a percentage of the face value of bond. Namely, we show that the defaultable zero-coupon bonds with stochastic volatility tend to be overpriced or underpriced in terms of parameters involved. The parameter values used to calculate are $a=0.2, \widetilde{a}=$ $0.3, R=0.8, t=0$, and $T=1$. Table 1 has three types as follows: the second row only has a stochastic volatility $K_{1}^{\epsilon}$ factor $\left(K_{2}^{\epsilon}=K_{3}^{\epsilon}=0\right)$, the third row solely has a stochastic volatility $K_{2}^{\epsilon}$ factor $\left(K_{1}^{\epsilon}=K_{3}^{\epsilon}=0\right)$, and the fourth row uniquely has a stochastic volatility $K_{3}^{\epsilon}$ factor $\left(K_{1}^{\epsilon}=K_{2}^{\epsilon}=0\right)$. We can see from Table 1 that the mispricing of the defaultable zero-coupon bond moves in the same direction from -0.07 to 0.07 with respect to each of the stochastic volatility terms. We also study the mispricing of the defaultable zero-coupon bond with respect to recovery rate. The same coefficients are used as for Table 1 except for $K_{1}^{\epsilon}=0.01, K_{2}^{\epsilon}=-0.03$, and $K_{3}^{\epsilon}=$ -0.02 . Table 2 shows that the mispricing of the defaultable zero-coupon bond monotonically decreases when the value of the recovery rate increases.

We report the defaultable bond price and the yield curve caused by changes in the value of time to maturity (source: KIS Pricing's). The parameter values used to calculate are $a=0.1, \widetilde{a}=0.2, \sigma=0.1, \bar{\sigma}(z)=0.16, \breve{\sigma}^{2}(z)=0.19, \rho_{r \lambda}=$ $-0.1, K_{1}^{\epsilon}=-0.06, K_{2}^{\epsilon}=0.02, K_{3}^{\epsilon}=-0.04, r=0.17, \lambda=$ 0.15 , and $R=0.3$ in Figures 1 and 2, respectively. Figures 1 and 2 have two cases of curve. Case 1 corresponds to the

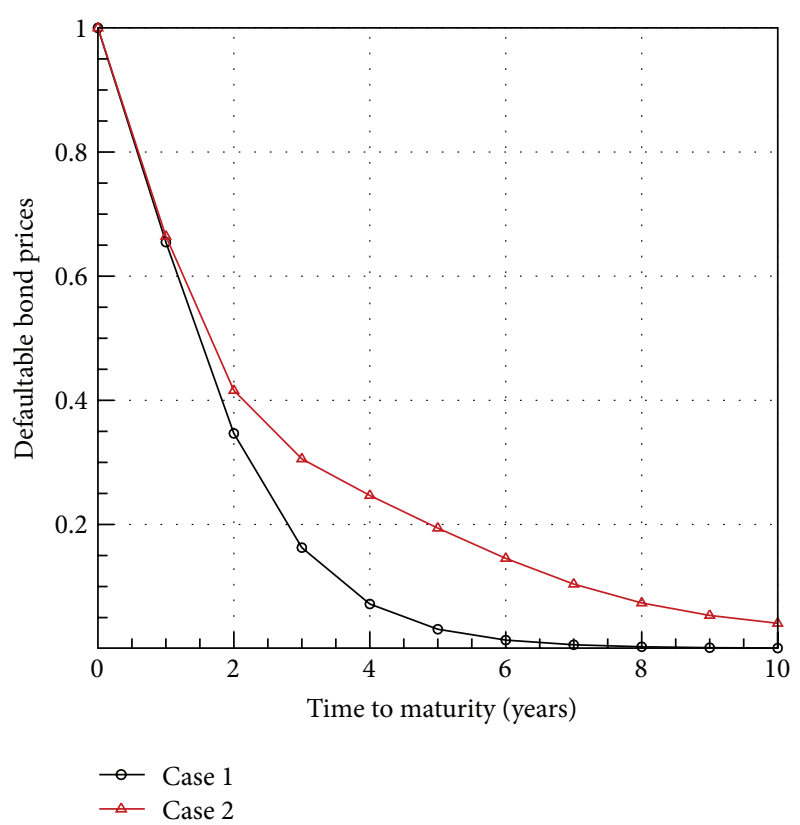

Figure 1: Defaultable zero-coupon bond prices.

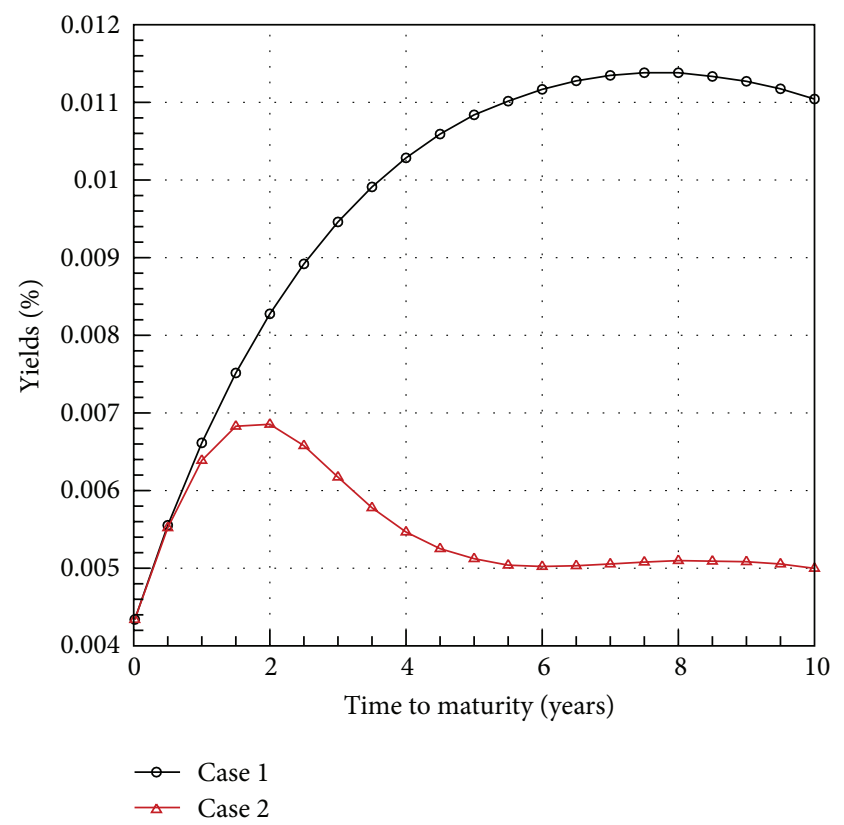

Figure 2: Yield curves.

constant volatility of the interest rate process. Case 2 contains the stochastic volatility of the interest rate process. Figure 1 shows that the defaultable bond prices with the stochastic intensity becomes higher than the defaultable bond prices with the constant volatility as the time to maturity increase. Also, the hump-shaped yield curve that matches a yield curve for structural model as in the Merton [3] appears to Case 2 in Figure 2. Hence, the stochastic volatility affects both quantitative and qualitative effect. 
TABLE 1: Effect of stochastic volatility terms.

\begin{tabular}{lcccccc}
\hline Stochastic volatility value & -0.07 & -0.05 & -0.01 & 0 & 0.01 & 0.05 \\
\hline $\begin{array}{l}\text { Mispricing as percentage of defaultable } \\
\text { zero-coupon bond price with respect to } K_{1}^{\epsilon}\end{array}$ & 0.051 & 0.037 & 0.007 & 0 & -0.007 & -0.037 \\
$\begin{array}{l}\text { Mispricing as percentage of defaultable } \\
\text { zero-coupon bond price with respect to } K_{2}^{\epsilon}\end{array}$ & 0.265 & 0.190 & 0.038 & 0 & -0.038 & -0.191 \\
$\begin{array}{l}\text { Mispricing as percentage of defaultable } \\
\text { zero-coupon bond price with respect to } K_{3}^{\epsilon}\end{array}$ & 1.364 & 0.978 & 0.197 & 0 & -0.002 & -0.010 \\
\hline
\end{tabular}

TABLE 2: Effect of recovery rate.

\begin{tabular}{lcccccc}
\hline Recovery rate $(R)$ & 0 & 0.2 & 0.4 & 0.6 & 0.8 & 1 \\
\hline $\begin{array}{l}\text { Mispricing as percentage of } \\
\text { defaultable zero-coupon } \\
\text { bond price }\end{array}$ & 0.78 & 0.72 & 0.67 & 0.59 & 0.49 & 0.39 \\
\hline
\end{tabular}

\section{Pricing Credit Default Swaps and Bond Options}

In this section, we get formulas for the CDS and the bond option by using the results of Section 3.

4.1. Pricing Credit Default Swap. A CDS is a bilateral contract in which one party (the protection buyer) pays a periodic, fixed premium to another (the protection seller) for protection related to credit events on an underlying reference entity. If a credit event occurs, the protection seller is obliged to make a payment to the protection buyer in order to compensate him for any losses that he might otherwise incur. Thus, the credit risk of the reference entity is transferred from the protection buyer to the protection seller. In particular, $\mathrm{Ma}$ and Kim [22] first study the problem of default correlation when the reference entity and the protection seller can occur default at the same time.

For simplicity, we suppose the following.

(i) We consider a forward CDS rate, valuable after some initial time $t_{0}$ with $0 \leq t_{0}<t_{1}$, for using the results of Section 3.

(ii) Let $M$ be the time-to-maturity of a forward CDS contract, $t_{1}<\cdots<t_{N}=M$ the premium payment dates, and $\mathscr{T}$ the payment tenor.

(iii) The bond coupon dates match the payment dates of the CDS.

(iv) If a credit event happens, then the settlement takes place at coupon date following default, but we do not consider the accrued premium payment.

The premium leg is the series of payments of the forward CDS rate until maturity or until the first default time $\tau$. Let the price $C\left(t, t_{0} ; \mathscr{T}\right)$ of the forward CDS rate and the price
$C^{\mathrm{pb}}\left(t, t_{0} ; \mathscr{T}\right)$ of the protection buyer paying $1_{(\tau>t)}$ at time $t$ is given by

$$
\begin{aligned}
& C^{\mathrm{pb}}\left(t, t_{0} ; \mathscr{T}\right) \\
& \quad=C\left(t, t_{0} ; \mathscr{T}\right) \mathbb{E}^{*} \sum_{n=1}^{N}\left[e^{-\int_{t}^{t_{n}}\left(r_{s}+\lambda_{s}\right) d s} \mid r_{t}=r, \lambda_{t}=\lambda, y_{t}=y\right] .
\end{aligned}
$$

Note that we can easily solve (34) by using (33) when $R=0$ (zero recovery).

On the other hand, let the price $C^{\mathrm{ps}}\left(t, t_{0} ; \mathscr{T}\right)$ of the protection seller at the default time $\tau$ is given by

$$
\begin{aligned}
& C^{\mathrm{ps}}\left(t, t_{0} ; \mathscr{T}\right) \\
& \quad=(1-R) \mathbb{E}^{*}\left[\int_{t_{0}}^{t_{N}} e^{-\int_{t}^{u}\left(r_{s}+\lambda_{s}\right) d s} \lambda_{u} d u \mid r_{t}=r, \lambda_{t}=\lambda, y_{t}=y\right],
\end{aligned}
$$

with $t<\tau$. Note that the protection seller payment is zero on $\{t \geq \tau\}$.

Finally, plugging (34) and (35), we obtain the forward CDS rate $C\left(t, t_{0} ; \mathscr{T}\right)$ as follows:

$$
\begin{aligned}
C( & \left.t, t_{0} ; \mathscr{T}\right) \\
= & (1-R) \\
& \times\left(\mathbb{E}^{*}\left[\int_{t_{0}}^{t_{N}} e^{-\int_{t}^{u}\left(r_{s}+\lambda_{s}\right) d s} \lambda_{u} d u \mid r_{t}=r, \lambda_{t}=\lambda, y_{t}=y\right]\right) \\
& \times\left(\sum_{n=1}^{N} \mathbb{E}^{*}\left[e^{-\int_{t}^{t_{n}}\left(r_{s}+\lambda_{s}\right) d s} \mid r_{t}=r, \lambda_{t}=\lambda, y_{t}=y\right]\right)^{-1},
\end{aligned}
$$

with $t<\tau$. Note that the spread of a CDS rate is given by the spread of a forward CDS when $t_{0}=t$. Refer to Ma and Kim [22].

Now, we will calculate (35) by using the asymptotic analysis. For the protection seller payment, we put

$$
\begin{aligned}
\breve{C} & (t, r, \lambda, y ; T) \\
& =\mathbb{E}^{*}\left[\int_{t}^{T} e^{-\int_{t}^{u}\left(r_{s}+\lambda_{s}\right) d s} \lambda_{u} d u \mid r_{t}=r, \lambda_{t}=\lambda, y_{t}=y\right], \\
& C^{\mathrm{ps}}\left(t, t_{0} ; \mathscr{T}\right)=\breve{C}\left(t, r, \lambda, y ; t_{N}\right)-\breve{C}\left(t, r, \lambda, y ; t_{0}\right) .
\end{aligned}
$$


Using the three-dimensional Feynman-Kac formula we have the following Kolmogorov PDE:

$$
\begin{aligned}
& \breve{\mathscr{L}} \breve{C}(t, r, \lambda, y ; T)=-\lambda, \quad t<T, \\
& \left.\breve{C}(t, r, \lambda, y ; T)\right|_{t=T}=0,
\end{aligned}
$$

where

$$
\begin{gathered}
\breve{\mathscr{L}}:=\frac{1}{\epsilon} \mathscr{L}_{0}+\frac{1}{\sqrt{\epsilon}} \mathscr{L}_{1}+\breve{\mathscr{L}}_{2}, \\
\breve{\mathscr{L}}_{2}=\frac{\partial}{\partial t}+\left(\theta_{t}-a r\right) \frac{\partial}{\partial r}+\left(\widetilde{\theta}_{t}-\tilde{a} \lambda\right) \frac{\partial}{\partial \lambda}+\frac{1}{2} \sigma^{2} \frac{\partial^{2}}{\partial r^{2}} \\
+\frac{1}{2} f^{2}(y) \frac{\partial^{2}}{\partial \lambda^{2}}+\sigma f(y) \rho_{r \lambda} \frac{\partial^{2}}{\partial r \partial \lambda}-(r+\lambda),
\end{gathered}
$$

$\mathscr{L}_{0}$ and $\mathscr{L}_{1}$ are defined by in Section 3 , respectively. $\breve{\mathscr{L}}_{2}$ corresponds to $\mathscr{L}_{2}$ with $R=0$ (zero recovery).

We consider an expansion of $\breve{C}$ in the following form:

$$
\breve{C}=\breve{C}_{0}+\sqrt{\epsilon} \breve{C}_{1}+\epsilon \breve{C}_{2}+\epsilon \sqrt{\epsilon} \breve{C}_{3}+\cdots
$$

Applying (40) to (38) leads to

$$
\begin{aligned}
\frac{1}{\epsilon} \mathscr{L}_{0} \breve{C}_{0} & +\frac{1}{\sqrt{\epsilon}}\left(\mathscr{L}_{0} \breve{C}_{1}+\mathscr{L}_{1} \breve{C}_{0}\right)+\left(\mathscr{L}_{0} \breve{C}_{2}+\mathscr{L}_{1} \breve{C}_{1}+\breve{\mathscr{L}}_{2} \breve{C}_{0}\right) \\
& +\sqrt{\epsilon}\left(\mathscr{L}_{0} \breve{C}_{3}+\mathscr{L}_{1} \breve{C}_{2}+\breve{\mathscr{L}}_{2} \breve{C}_{1}\right)+\cdots=0 .
\end{aligned}
$$

Through similar processes in Section 3, we will calculate the leading order term $\breve{C}_{0}$ and the first perturbation term $\breve{C}_{1}$. Namely, we let

$$
\left\langle\breve{\mathscr{L}}_{2}\right\rangle \breve{C}_{0}=-\lambda \text {, }
$$

with the terminal condition $\left.\breve{C}_{0}(t, r, \lambda ; T)\right|_{t=T}=0$ and

$$
\left\langle\breve{\mathscr{L}}_{2}\right\rangle \bar{C}_{1}=\breve{\mathscr{A}}_{0}
$$

with the terminal condition $\left.\bar{C}_{1}(t, r, \lambda ; T)\right|_{t=T}=0$, where $\bar{C}_{1}:=$ $\sqrt{\epsilon} \breve{C}_{1}$ and $\breve{\mathscr{A}}:=(1 / \sqrt{\alpha})\left\langle\mathscr{L}_{1} \mathscr{L}_{0}^{-1}\left(\breve{\mathscr{L}}_{2}-\left\langle\breve{\mathscr{L}}_{2}\right\rangle\right)\right\rangle$.

Let $P_{0}(t, r, \lambda ; T)$ be equal to the leading order term $P_{0}(t, r, \lambda ; T)$ in (13) of the approximate solution of a defaultable zero-coupon bond for zero recovery, that is,

$$
P_{0}(t, r, \lambda ; T)=e^{A(t, T)-B(t, T) r-C(t, T) \lambda},
$$

with $A(T, T)=B(T, T)=C(T, T)=0$, where $A(t, T), B(t, T)$ and $C(t, T)$ are given by $(14)$.

Hence, the solutions of (42) and (43) are

$$
\begin{gathered}
\breve{C}_{0}(t, r, \lambda ; T)=\lambda \int_{t}^{T} P_{0}(s, r, \lambda ; T) d s, \\
\bar{C}_{1}(t, r, \lambda ; T)=\lambda \int_{t}^{T} \int_{s}^{T}\left[K_{1}^{\epsilon} B(s, h) C^{2}(s, h)\right. \\
+K_{2}^{\epsilon} B^{2}(s, h) C(s, h) \\
\left.+K_{3}^{\epsilon} C^{3}(s, h)\right] P_{0}(s, r, \lambda ; h) d h d s,
\end{gathered}
$$

respectively.
Therefore, synthesizing (45), we derive an asymptotic analysis of the protection seller at time $t$ which is given by

$$
\breve{C}(t, r, \lambda, y ; T) \approx \breve{C}_{0}(t, r, \lambda ; T)+\bar{C}_{1}(t, r, \lambda ; T) .
$$

4.2. Bond Option Pricing. In this subsection, we obtain an asymptotic option pricing formula when the underlying asset itself is a defaultable zero-coupon bond.

Let $T_{0}$ be the maturity of the option and $T$ be the maturity of the defaultable zero-coupon bond with $T_{0}<T$. We assume that the option becomes invalid when a default occurs prior to $T_{0}$ and that the defaultable zero-coupon bond has the fractional recovery $R$ as before. The option price at time $t$ for an interest rate process $r_{t}=r$, an intensity process $\lambda_{t}=$ $\lambda$, and a stochastic volatility process $y_{t}=y$, denoted by $U\left(t, r, \lambda, y ; T_{0}, T\right)$, is given by

$$
\begin{aligned}
& U\left(t, r, \lambda, y ; T_{0}, T\right) \\
& \quad=E^{*}\left[e^{-\int_{t}^{T_{0}} r_{s} d s} l\left(\mathscr{B}\left(T_{0}, T\right)\right) \mid r_{t}=r, \lambda_{t}=\lambda, y_{t}=y\right],
\end{aligned}
$$

under the martingale measure $P^{*}$, where the bond price $\mathscr{B}\left(T_{0}, T\right)$ is

$$
\begin{aligned}
\mathscr{B}\left(T_{0}, T\right) & :=P\left(T_{0}, r_{T_{0}}, \lambda_{T_{0}}, y_{T_{0}} ; T\right) \\
& =E^{*}\left[e^{-\int_{T_{0}}^{T}\left(r_{s}+(1-R) \lambda_{s}\right) d s} \mid r_{T_{0}}, \lambda_{T_{0}}, y_{T_{0}}\right],
\end{aligned}
$$

and $l\left(\mathscr{B}\left(T_{0}, T\right)\right)$ is the payoff function of the option at time $T_{0}$. It is assumed that the payoff function $h$ is at the best linearly growing at infinity and is a smooth function. This smoothness assumption may be too severe in practical point of view as $h$ is not differentiable at the exercise price in the classical European call or put option case. In fact, the smoothness assumption on $h$ can be removed as shown in Fouque et al. [27]. We take, however, the smoothness assumption for the simplicity of our argument here.

The three-dimensional Feynman-Kac formula corresponding to the price function $U$ is the same as the one for the defaultable zero-coupon bond in (9) but with a terminal condition, namely,

$$
\begin{gathered}
\mathscr{L} U\left(t, r, \lambda, y ; T_{0}\right)=0, \quad t<T_{0}, \\
U\left(T_{0}, r_{T_{0}}, \lambda_{T_{0}}, y_{T_{0}} ; T\right)=l\left(P\left(T_{0}, r_{T_{0}}, \lambda_{T_{0}}, y_{T_{0}} ; T\right)\right),
\end{gathered}
$$

where $\mathscr{L}$ is defined in (9). We take the expansions

$$
\begin{gathered}
U=U_{0}+\sqrt{\epsilon} U_{1}+\epsilon U_{2}+\cdots, \\
U\left(T_{0}, r_{T_{0}}, \lambda_{T_{0}}, y_{T_{0}} ; T\right) \\
=l\left(P_{0}\left(T_{0}, r_{T_{0}}, \lambda_{T_{0}} ; T\right)\right) \\
+\widetilde{P}_{1}\left(T_{0}, r_{T_{0}}, \lambda_{T_{0}} ; T\right) l^{\prime}\left(P_{0}\left(T_{0}, r_{T_{0}}, \lambda_{T_{0}} ; T\right)\right)+\cdots,
\end{gathered}
$$

where $P_{0}$ and $\widetilde{P}_{1}$ are given by Theorems 1 and 2 , respectively. 
Using the similar argument as in Section 3, the terms of order $1 / \epsilon$ and $1 / \sqrt{\epsilon}$ in the asymptotic PDE of (9) provide the $y$-independence of $U_{0}$ and $U_{1}$.

The order-1 terms give a Poisson equation in $U_{2}$ from which the solvability condition $\left\langle\mathscr{L}_{2}\right\rangle U_{0}=0$ is satisfied. From (49) the corresponding terminal condition is given by $\left.U_{0}\right|_{t=T_{0}}=l\left(P_{0}\left(T_{0}, r_{T_{0}}, \lambda_{T_{0}} ; T\right)\right)$. Then, as calculated in Section 3.1, we have

$$
\begin{aligned}
& U_{0}\left(t, r, \lambda ; T_{0}, T\right) \\
& \quad=E^{*}\left[e^{-\int_{t}^{T_{0}} r_{s} d s} l\left(P_{0}\left(T_{0}, r_{T_{0}}, \lambda_{T_{0}} ; T\right)\right) \mid r_{t}=r, \lambda_{t}=\lambda\right],
\end{aligned}
$$

where $P_{0}\left(T_{0}, r_{T_{0}}, \lambda_{T_{0}} ; T\right)$ is given by Theorem 1 at time $t=T_{0}$.

The order- $\sqrt{\epsilon}$ terms lead to a Poisson equation in $U_{3}$ with the solvability condition $\left\langle\mathscr{L}_{1} U_{2}+\mathscr{L}_{2} U_{1}\right\rangle=0$. If we put $\widetilde{U}_{1}:=$ $\sqrt{\epsilon} U_{1}$, then this solvability condition leads to the following PDE:

$$
\left\langle\mathscr{L}_{2}\right\rangle \widetilde{U}_{1}\left(t, r, \lambda ; T_{0}, T\right)=\mathscr{A} U_{0}\left(t, r, \lambda ; T_{0}, T\right),
$$

with the terminal condition given by

$$
\begin{aligned}
\widetilde{U}_{1} & \left(T_{0}, r_{T_{0}}, \lambda_{T_{0}} ; T_{0}, T\right) \\
& =\widetilde{P}_{1}\left(T_{0}, r_{T_{0}}, \lambda_{T_{0}} ; T\right) l^{\prime}\left(P_{0}\left(T_{0}, r_{T_{0}}, \lambda_{T_{0}} ; T\right)\right),
\end{aligned}
$$

from (50), where the operator $\mathscr{A}$ and $\widetilde{P}_{1}$ are given by (30) and Theorem 2, respectively. Then, by the Feynman-Kac formula applied to (52) and (53), we obtain the following probabilistic representation:

$$
\begin{aligned}
& \widetilde{U}_{1}\left(t, r, \lambda ; T_{0}, T\right) \\
& =E^{*}\left[e^{-\int_{t}^{T_{0}} r_{s} d s} \widetilde{P}_{1}\left(T_{0}, r_{T_{0}}, \lambda_{T_{0}} ; T\right) l^{\prime}\left(P_{0}\left(T_{0}, r_{T_{0}}, \lambda_{T_{0}} ; T\right)\right)\right. \\
& \left.\quad-\int_{t}^{T_{0}} e^{-\int_{t}^{u} r_{s} d s} \mathscr{A} U_{0}\left(u, r, \lambda ; T_{0}, T\right) d u \mid r_{t}=r, \lambda_{t}=\lambda\right] .
\end{aligned}
$$

Synthesizing the above results, we obtain an asymptotic expansion of the defaultable zero-coupon bond option price as follows:

$$
U\left(t, r, \lambda, y ; T_{0}, T\right) \approx U_{0}\left(t, r, \lambda ; T_{0}, T\right)+\widetilde{U}_{1}\left(t, r, \lambda ; T_{0}, T\right),
$$

where $U_{0}$ and $\widetilde{U}_{1}$ are given by (51) and (54), respectively.

\section{Final Remarks}

In this paper, we consider the intensity-based defaultable bonds of two-correlated-factor Hull-White model with stochastic volatility of interest rate process. Using the asymptotic analysis, developed by Fouque et al. [25], we get approximate solutions to price the defaultable zero-coupon bonds as well as the credit default swaps and the bond options. It shows how these small perturbations can affect the shape of the yield curve of the defaultable zero-coupon bond. For further study, one can apply the framework of this paper to the multiname intensity models studied by Ma and Kim [28].

\section{References}

[1] D. Duffie and K. J. Singleton, Credit Risk, Princeton University Press, 2003.

[2] T. R. Bielecki and M. Rutkowski, Credit Risk: Modelling, Valuation and Hedging, Springer, New York, NY, USA, 2004.

[3] R. C. Merton, "On the pricing of corporate debt: the risk structure of interest rates," Journal of Finance, vol. 29, no. 2, pp. 449-470, 1974.

[4] F. A. Longstaff and E. S. Schwartz, "A simple approach to valuing risky fixed and floating rate," Journal of Finance, vol. 50, no. 3, pp. 789-819, 1995.

[5] H. E. Leland and K. B. Toft, "Optimal capital structure, endogenous bankruptcy, and the term structure of credit spreads," Journal of Finance, vol. 51, no. 3, pp. 987-1019, 1996.

[6] C. Zhou, "The term structure of credit spreads with jump risk," Journal of Banking and Finance, vol. 25, no. 11, pp. 2015-2040, 2001.

[7] D. Duffie and D. Lando, "Term structures of credit spreads with incomplete accounting information," Econometrica, vol. 69, no. 3, pp. 633-664, 2001.

[8] B. Hilberink and L. C. G. Rogers, "Optimal capital structure and endogenous default," Finance and Stochastics, vol. 6, no. 2, pp. 237-263, 2002.

[9] K. Giesecke, "Correlated default with incomplete information," Journal of Banking and Finance, vol. 28, no. 7, pp. 1521-1545, 2004.

[10] R. A. Jarrow and S. M. Turnbull, "Pricing derivatives on financial securities subject to credit risk," Journal of Finance, vol. 50, no. 1, pp. 53-85, 1995.

[11] D. B. Madan and H. Unal, "Pricing the risks of default," Review of Derivatives Research, vol. 2, no. 2-3, pp. 121-160, 1998.

[12] D. Lando, "On cox processes and credit risky securities," Review of Derivatives Research, vol. 2, no. 2-3, pp. 99-120, 1998.

[13] D. Duffie and K. J. Singleton, "Modeling term structures of defaultable bonds," Review of Financial Studies, vol. 12, no. 4, pp. 687-720, 1999.

[14] P. Collin-Dufresne and R. S. Goldstein, "Do bonds span the fixed income markets? Theory and evidence for unspanned stochastic volatility," Journal of Finance, vol. 57, no. 4, pp. 16851730, 2002.

[15] P. J. Schönbucher, "Term structure modelling of defaultable bonds," Review of Derivatives Research, vol. 2, no. 2-3, pp. 161$192,1998$.

[16] L. Tchuindjo, "Pricing of multi-defaultable bonds with a twocorrelated-factor Hull-White model," Applied Mathematical Finance, vol. 14, no. 1, pp. 19-39, 2007.

[17] G. R. Duffee, "Estimating the price of default risk," Review of Financial Studies, vol. 12, no. 1, pp. 197-226, 1999.

[18] J. Driessen, "Is default event risk priced in corporate bonds?" Review of Financial Studies, vol. 18, no. 1, pp. 165-195, 2005.

[19] E. Bayraktar and B. Yang, "A unified framework for pricing credit and equity derivatives," Mathematical Finance, vol. 21, no. 3, pp. 493-517, 2011.

[20] D. Duffie and N. Gârleanu, "Risk and valuation of collateralized debt obligations," Financial Analysts Journal, vol. 57, no. 1, pp. 41-59, 2001.

[21] E. Papageorgiou and R. Sircar, "Multiscale intensity models for single name credit derivatives," Applied Mathematical Finance, vol. 15, no. 1-2, pp. 73-105, 2008. 
[22] Y.-K. Ma and J.-H. Kim, "Pricing the credit default swap rate for jump diffusion default intensity processes," Quantitative Finance, vol. 10, no. 8, pp. 809-817, 2010.

[23] P. Cotton, J.-P. Fouque, G. Papanicolaou, and R. Sircar, "Stochastic volatility corrections for interest rate derivatives," Mathematical Finance, vol. 14, no. 2, pp. 173-200, 2004.

[24] J. Hull and A. White, "Pricing interest-rate derivative securities," Review of Financial Studies, vol. 3, pp. 573-592, 1990.

[25] J.-P. Fouque, G. Papanicolaou, R. Sircar, and K. Sølna, Multiscale Stochastic Volatility for Equity, Interest Rate, and Credit Derivatives, Cambridge University Press, Cambridge, UK, 2011.

[26] B. Øksendal, Stochastic Differential Equations, Springer, New York, NY, USA, 2003.

[27] J.-P. Fouque, G. Papanicolaou, R. Sircar, and K. Solna, "Multiscale stochastic volatility asymptotics," Multiscale Modeling \& Simulation, vol. 2, no. 1, pp. 22-42, 2003.

[28] Y.-K. Ma and J.-H. Kim, "Joint survival probability using truncated invariant farlie-gumbel-morgenstern copula," Working paper. 


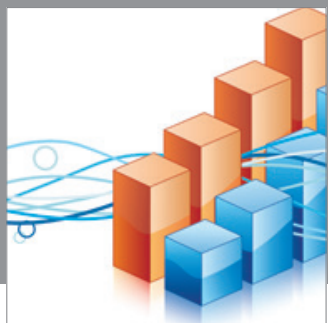

Advances in

Operations Research

mansans

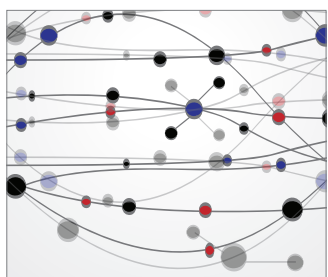

The Scientific World Journal
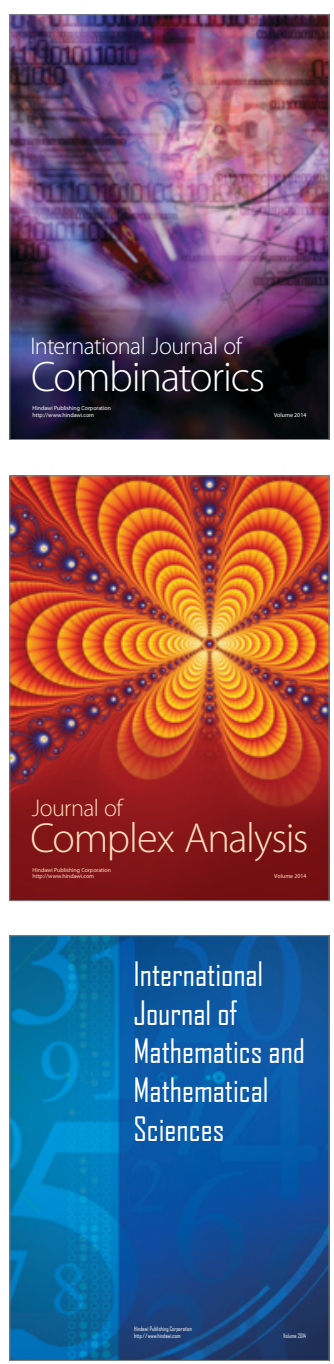
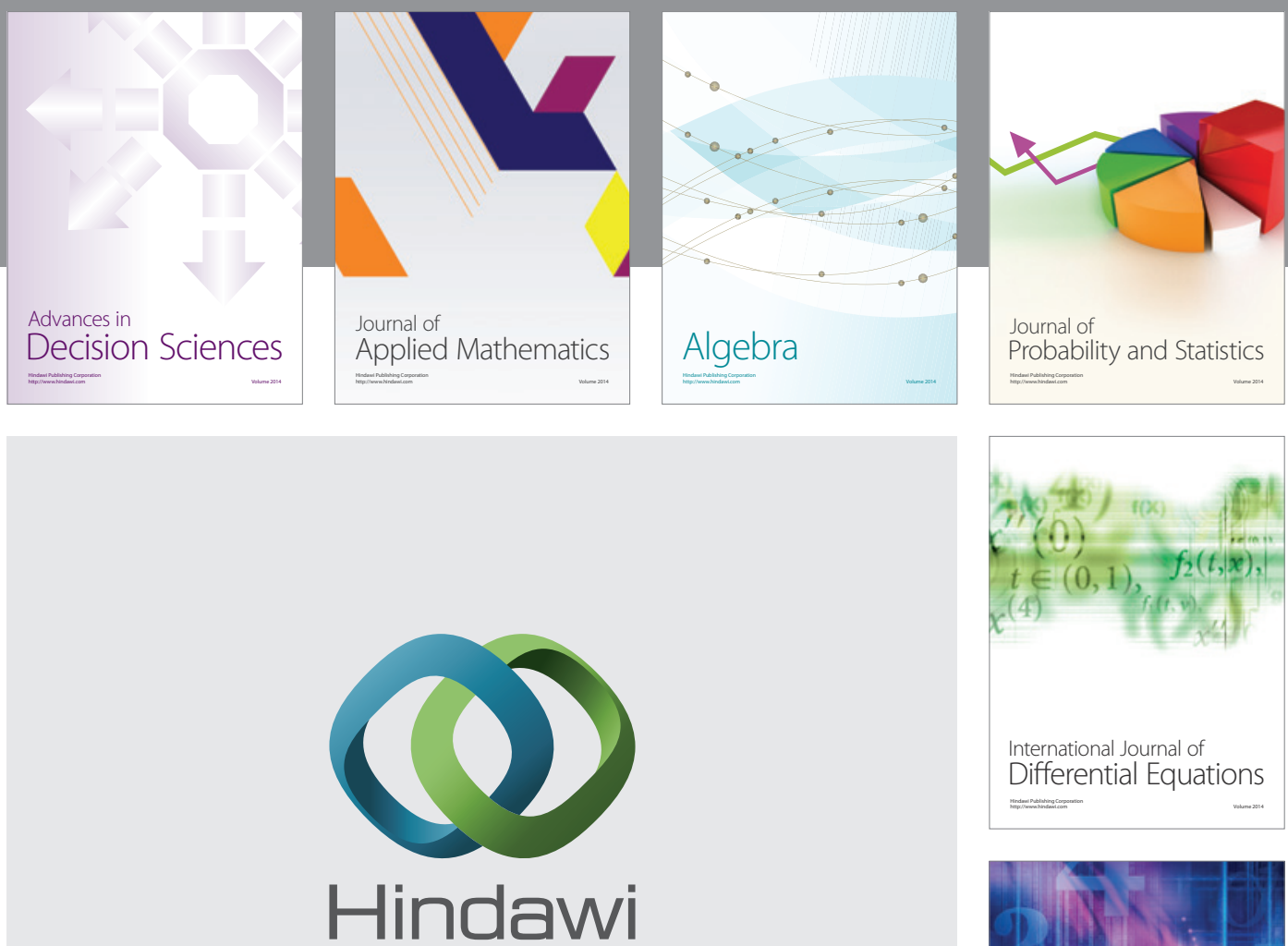

Submit your manuscripts at http://www.hindawi.com
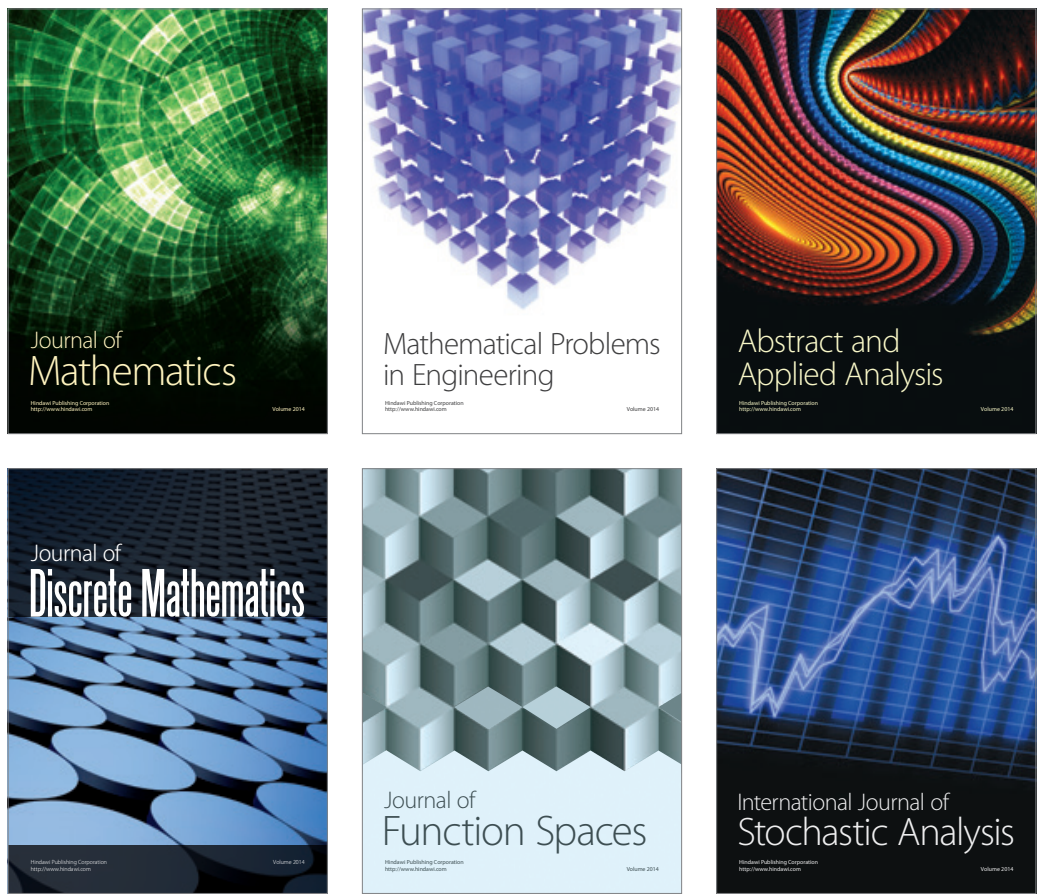

Journal of

Function Spaces

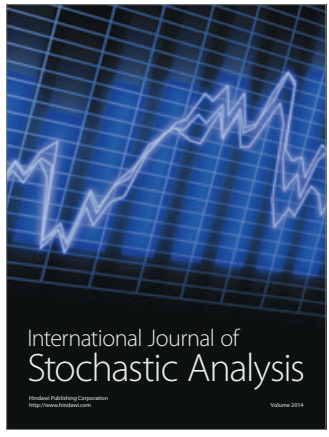

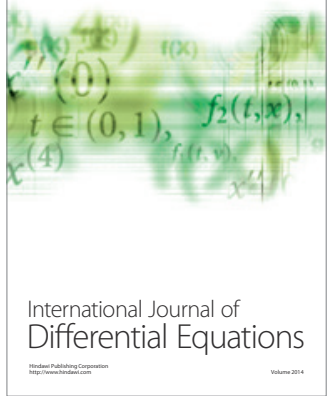
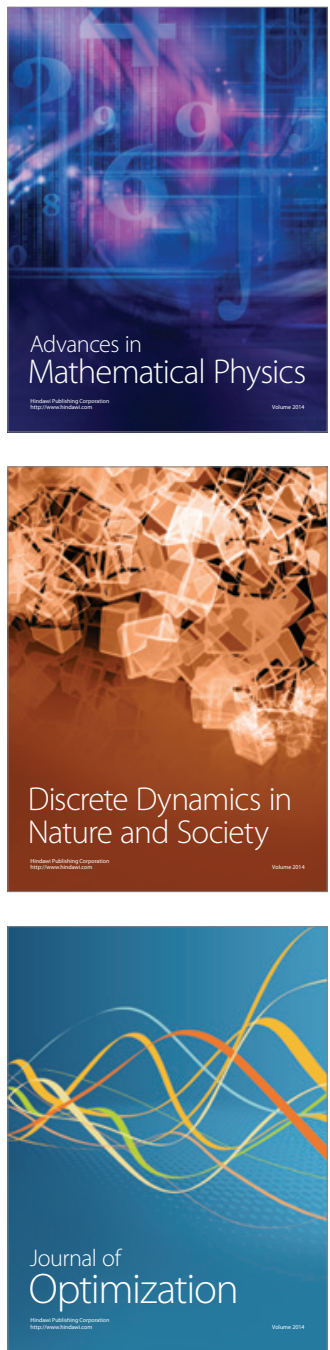